\title{
How the Usability of a Pen-Tablet System Varies with Response Time Retardation
}

\author{
Kei Teramoto, Shigeki Kuwata, and Hiroshi Kondoh \\ Division of Medical Informatics, Tottori University Hospital \\ 36-1 Nishi-cho, Yonago, Tottori, 683-8504, Japan \\ \{kei, shig, kondoh\} @med. tottori-u.ac.jp
}

\begin{abstract}
To realize cloud computing (CC), virtualization technology has been accepted as an infrastructure of Electronic Patient Records (EPR). However, it has not been sufficiently done so far to evaluate the usability of Pen-Tablet Systems (PTS) as an input device of EPR with CC environments. From our preliminary studies, we confirmed that response time retardation, which virtualization technology inherently bears, would be an influencing factor for the usability. In this study we conducted usability questionnaires for subjects who were requested to use a drawing application that produced the pseudo retardation in order to measure an allowable range on the retardation for implementing PTS on EPR with CC environments. Usability of the PTS would drastically drop with increasing values of the scattering of delays exceeding 35 milliseconds. As a result, this study would provide useful measures for implementing PTS with CC technology.
\end{abstract}

Keywords: Cloud Computing, Pen-Tablet System, Usability.

\section{Introduction}

Thin-client computing, well known as cloud computing (CC) or Server-Based computing, for hospital information system has been widely accepted, because the technology is able to decrease the cost of ownership and enhance a security level [1]. However, it has not been sufficiently done so far to evaluate the usability of PenTablet Systems (PTS) as an input device of Electronic Patient Records (EPR) with CC environments. This is a comparative study on the usability of a standalone PC and a thin-client as a terminal with CC technology when users utilize PTS with EPR. Response time retardation, often occurring in CC environments, was evaluated in terms of the degrees of its impact on the usability by an experiment using an application to produce the controlled pseudo retardation (delay) of response time.

\section{Method}

From the preliminary studies, we confirmed that the response time retardation varied with virtualization software for implementing EPR on CC environments [2]. In the current experiment, five subjects with the age ranging from 28 to 40 having over 10 
years of PC experience were requested to write signatures using PTS with a drawing application, subsequently to answer three-grade questionnaires on the usability (5: equal to standalone PC, 3: inferior but usable and 1: not usable). The drawing application controlled an average of the delays increased from 0 to 120 milliseconds (msec) with the interval of $20 \mathrm{msec}$, as well as a standard deviation of the delays increased from 28 to 80 with the interval of approximately $3 \mathrm{msec}$.

\section{Results and Conclusion}

As a result, we found that the scattering, as a standard deviation of the delays, would impact on use of PTS much more than the delay length, as an average of the delays. Since the result was also supported by our preliminary studies [3], it is reasonable to suppose that the scattering can be a useful indicator for using PTS on CC environment.

In terms of the relationship between the scattering of the delays and the usability evaluated by subjects, the best usability was obtained for subjects under the condition of the delay length within $100 \mathrm{msec}$ and the scattering within $35 \mathrm{msec}$ : the subjects marked approximately over 4 points on the usability questionnaires. When the scattering lay between 36 and $40 \mathrm{msec}$, the best usability was also found only with a very limited range of the delay length less than within $20 \mathrm{msec}$. When the scattering exceeded $40 \mathrm{msec}$, the results of evaluation showed the inferior usability even if PTS had no delays.

As conclusion, it would be desirable that values of the scattering range within 35 msec for using PTS with CC technology. Otherwise the usability would drastically drop with increasing values of the scattering, even if the values increased by only a few milliseconds.

Although this study would provide useful measures for implementing PTS, its applicability to hospital settings still remains limited: the number of subjects and the types of applications for virtualization were not sufficient in this study. Those limitations should be reexamined in further studies.

\section{References}

1. Kuwata, S., Teramoto, K., Yasushi, M., Kushniruk, A., Borycki, E., Kondo, H.: Effective Solutions in Introducing Server-Based Computing into Hospital Information System. Stud. Health Technol. Inform. 143, 435-440 (2009)

2. Teramoto, K., Kuwata, S., Kushniruk, A., Borycki, E., Kondo, H.: Issues on evaluating the usability of a pen-tablet system using server-based computing. Stud. Health Technol. Inform. 150, 415 (2009)

3. Teramoto, K., Kuwata, S., Kondoh, H.: Evaluation of the Usability of a Pen Tablet System Using Server-Based Computing: Evaluation of the Usability of a Pen Tablet System Using Server-Based Computing. In: Asia Pacific Association for Medical Informatics 2009, Lighthouse, Japan, vol. 27, pp. 182-186 (2009) 